\title{
NANOTECHNOLOGY IN DIAGNOSTICS AND DRUG DELIVERY
}

\author{
Hwa A. Lim, Ph.D., MBA \\ D’Trends, Inc., 3521 Ryder Street, Santa Clara, California 95051, USA \\ hal@d-trends.com
}

\begin{abstract}
The current state of nanotechnology is comparable with the level of technological development in polymers and plastics in the 1930s. At that time, the polymer and plastic industry was in its nascent stage but the industry quickly grew, providing the materials for a large portion of manufactured goods. Nanotechnology industry is currently in an equivalent infant stage, but several basic breakthroughs have been made. ${ }^{1}$ Based on the polymer and plastic analogy, the annual market for products that carry nano-components, including all computer chips, half of pharmaceuticals and half of chemical catalysts, will reach $\$ 1$ trillion by 2015 . Nanotechnology is thus expected to thoroughly affect the way science addresses medicine, food, electronics and the environment. ${ }^{2}$

In this article I will take a cursory look at the basic concepts of nanotechnology, outline unique properties of certain nano-materials, and illustrate how these properties can be utilized to overcome current technological bottlenecks in disease detection systems for diagnostic purposes and in drug delivery systems for therapeutic purposes.
\end{abstract}

Keywords: nanotechnology, nanobiotechnology, diagnostics, drug delivery, nanocrystals, hepatitis $\mathrm{B}$ virus.

\section{INTRODUCTION}

In science fiction movies we have seen miniaturization of humans, organisms and objects to miniscule sizes. As early as 1957 in "The Incredible Shrinking Man” flick, Scott Carey, after having been exposed to radioactivity, shrank to atomic size. In 1959, the famed Nobel laureate Richard Feynman made the by-now heavily cited statement "The principles of physics, as far as I can see, do not speak against the possibility of maneuvering things atom by atom. It is not an attempt to violate any laws; it is something, in principle, that can be done; but in practice, it has not been done because we are too big." ${ }^{\text {3 }}$ In 1966 in the science fiction book "Fantastic Voyage" a midget submarine swam in the human circulatory system to destroy a life-threatening clot. ${ }^{4}$ 
Recently more books, including "Prey" by Michael Crichton about evolution and nanomachines, and movies are being made of the technology. ${ }^{5}$

The prefix "nano" is actually derived from an ancient Greek word "nano" which means dwarf. In its present usage, nanoscience is the study of materials and events at the level of $10^{-9} \mathrm{~m}$, a billionth of a meter. It: is the domain where the sciences: physics, chemistry and biology, meet.

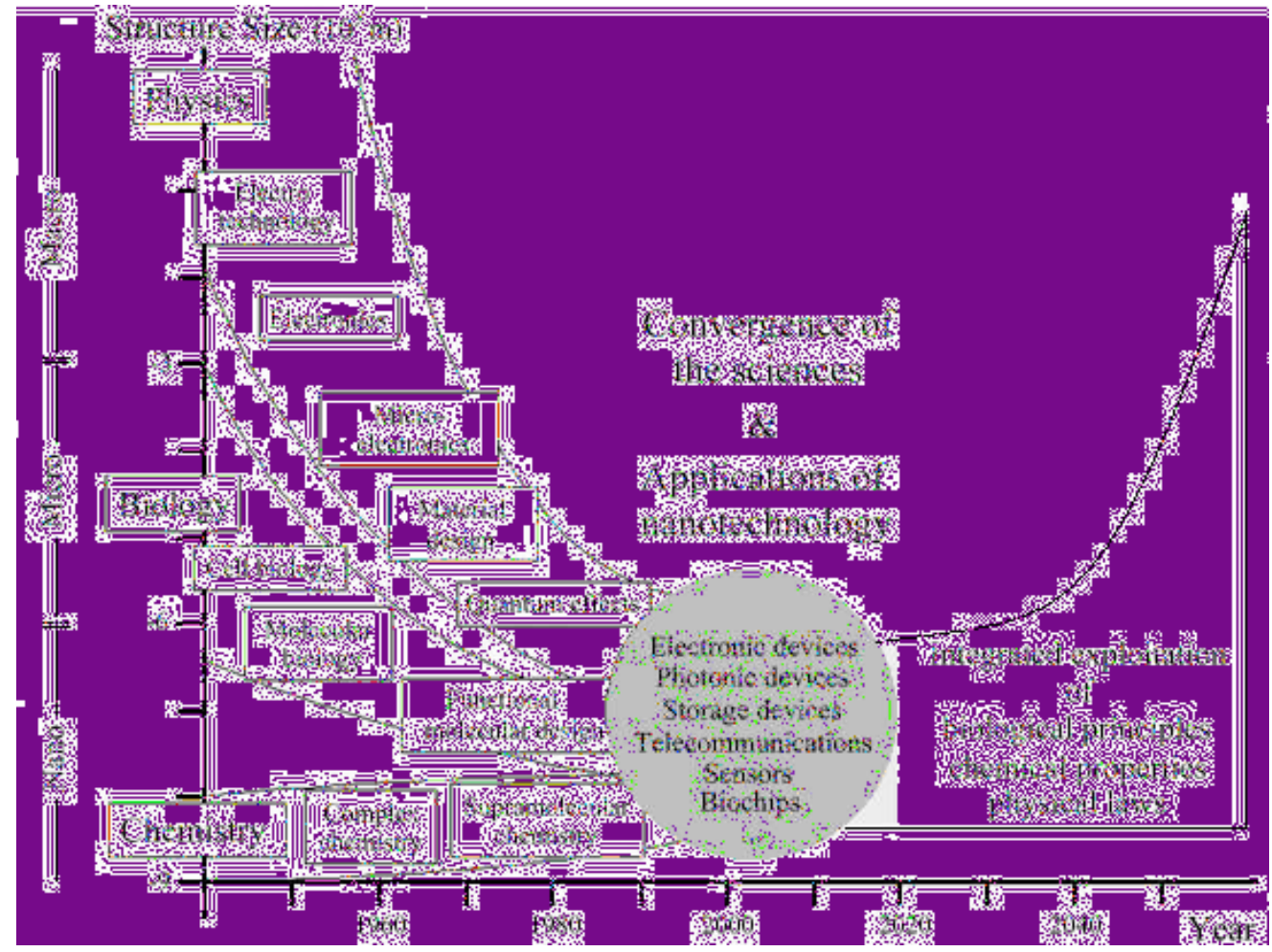

Figure 1. Nanoscience is where biology, chemistry and physics meet. (This figure is adapted from VDI Technology Center, Future Technologies Division).

To have a feel of how "dwarf" a nanometer is, it is equivalent to the width of ten hydrogen atoms laid side by side, or one-thousandth the length of a typical bacterium, or a fifty-thousandth the width of a human hair, or one-millionth the size of a pinhead. By definition, nanotechnology refers to research and development on structures that have at least one of the dimensions less than 100 nanometers. Thus a nanowire can be long, but its width is only 70nm; a DNA can be a 
long twisted pair, but the width is only $2-5 \mathrm{~nm}$; while a nanocrystal is $2-6 \mathrm{~nm}$ in diameter. Two salient features of nanotechnology are common:

q Creation of functional materials, devices and systems through the manipulations of matters at the nano-scale, which is in the range of $\sim 0.1-100 \mathrm{~nm}$,

q Exploitation of new and unique properties of materials such as nanopowders and nanocrystals, in which case the nano-scale is in the range of $\sim 1-100 \mathrm{~nm}$.

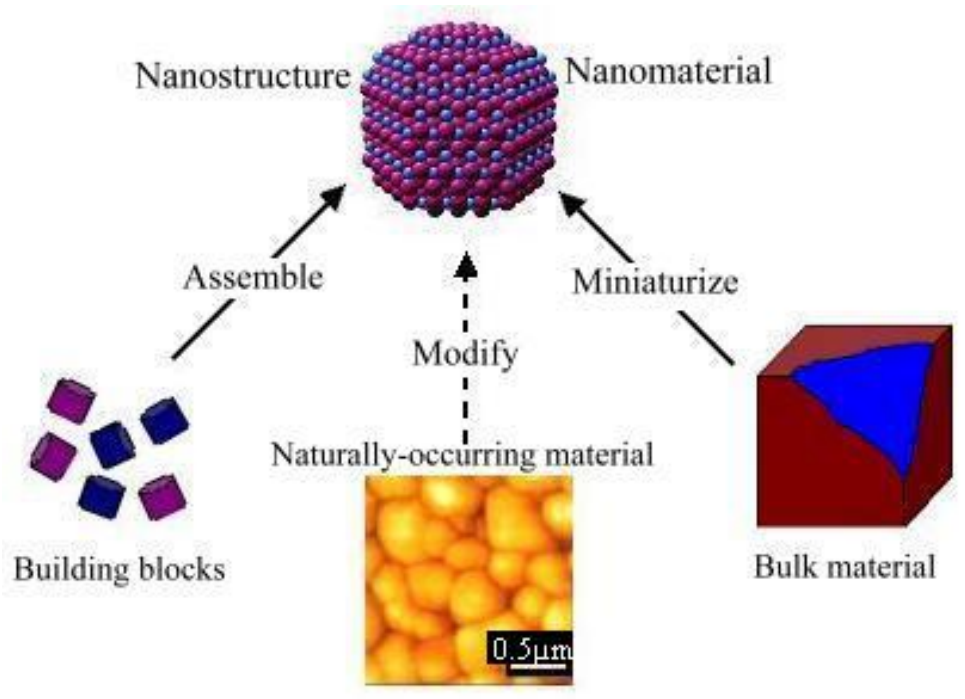

Figure 2. Three possible ways to get to the nano-scale.

In the first way, we start with a large block of bulk material and make it smaller by a certain process, for example, grinding titanium dioxide $\left(\mathrm{TiO}_{2}\right)$ into an ultra-fine powder of nano-size. The problem with this way of miniaturization is that the process is very wasteful.

In certain sense, nanotechnology and nanomaterials have been around since the first living organism inhabited the earth. Nature has always been based on atomic, nano-scaled architecture and nanomachines such as proteins, DNA, RNA, and other life-sustaining structures. This gives rise to the second way to work in the nano-domain - we make use of naturally occurring materials such as proteins and antibodies, which are already in the nano-size regime. The 
process is to modify these naturally occurring nanomachines to function in the way we want them to behave.

In yet a third way, we assemble subnano- and nano-size building blocks such as atoms or molecules exactly where we want them to be. The process is to position the constituent subnano building blocks into an assembly of a functional structure. The aforementioned Richard Feynman's 1959 statement connotes this process. The problem with this way of getting nano-size materials, with the current state of the art of technologies, is that the process is very time-consuming and expensive.

As the polymer and plastic industry lesson has shown us, with new nanotechnological breakthroughs and ways to bring the cost down, there is no reason why the nanotechnology industry will not rise to similar, if not greater, prominence.

\section{IMPORTANCE AND POTENTIAL ECONOMICAL IMPACT}

At the nano-scale, properties and behaviors of everyday material change significantly. Nano-size particles exhibit size-dependent properties that are profoundly different from the corresponding bulk material. Other physical, chemical and optical properties that change drastically when the physical dimension of a material approaches the nano range include:

Table 1. Properties of material change drastically at the nano-scale. (This table is adapted from John Werry,

"Nanotechnology and you").

\begin{tabular}{|l|l|}
\hline Electrical & $\begin{array}{l}\text { Higher electrical conductivity in ceramics and magnetic nanocomposites; higher resistivity in } \\
\text { metals. }\end{array}$ \\
\hline Magnetic & $\begin{array}{l}\text { Increase in magnetic coercivity down to a critical size in the nano regime; below the critical } \\
\text { crystalline size, decrease in coercivity leads to superparamagnetic behavior. }\end{array}$ \\
\hline Mechanical & $\begin{array}{l}\text { Increase in hardness and strength of metals and alloys; enhanced ductility, toughness and } \\
\text { formability of ceramics; super strength and super plasticity. }\end{array}$ \\
\hline Optical & Increase in luminescent efficiency of semiconductors; transparency of nanoparticles. \\
\hline Chemical & Substantial increase in catalytic properties and reaction rates. \\
\hline
\end{tabular}


Not surprisingly, many organizations in the public and private sectors are already heavily involved in the research and development of nanotechnology.

Table 2. Based on 150 companies, the relative percentages of nano efforts in various areas are as follows. (This table is adapted from NanoBusiness Alliance).

\begin{tabular}{lr}
\hline Area & Percentage \\
\hline Materials, production techniques & 31 \\
Medicine, pharmaceutics & 21 \\
Research & 14 \\
Electronics & 11 \\
Consumer products & 7 \\
Energy storage & 4 \\
IT & 4 \\
Nano-analysis & 4 \\
Telecommunications & 4 \\
\hline
\end{tabular}

The National Science \& Technology Council (NSTC) of United States estimates that the market size of nano-related material will reach $\$ 1$ trillion by 2015 . Of this, about $\$ 180$ billion will be in life sciences. To put the whole picture in proper perspective, we recall from various estimates that biotechnology generated some $\$ 26.5$ billion in 2000 , the worldwide in vitro diagnostics market is $\$ 19$ billion and is growing at $11 \%$. By the mid term of 2007 , this will have compounded to some $\$ 40$ billion. When biotechnology and nanotechnology are integrated into nanobiotechnology, the future market size is expected to be larger.

\section{APPLICATIONS IN NANOBIOTECHNOLOGY}

There have been a number of breakthroughs in nanotechnology. For example, recently the nanotech group of the University of Texas at Dallas, led by Ray Baughman, succeeded in weaving nanofibers, four times tougher than spider silk, to a few hundred meters long. ${ }^{6}$ Instead of providing a list of major breakthroughs, it suffices to note that many major applications of 
nanotechnology are in biotechnology, in the integrated field called nanobiotechnology. Here I will just provide two exemplary applications.

In the current heightened state of chemical and biological attacks, bioterrorism is the first thing that comes to mind when we talk about a detection system. But fortunately or unfortunately, bioterror prevention is not a high-volume business and in itself will not drive the analysis market, even if it underwrites research and development, primarily in the U.S. The big money is in common diseases and ailments. For example, there are millions of annual ER visits for respiratory infections alone. A lot of the viruses are hard to detect because they have to be cultured, a process that may take days, and the lab procedure may take three roomful of equipment of twelve feet square each. In contrast, a tiny chip that could detect 10 or 20 of the most common infections in real time or provide quick-turnaround tests would help in deciding whether to give the patients antibiotics or just send them home to bed.

After diagnostics and in the case that an ailment has been identified, therapeutics follows. Many of the existing treatment modalities are inherently limited by toxic side effects following systemic administration. There is thus an urgent need to develop more efficient and safe delivery systems for genes, drugs or proteins to treat and cure diseases.

In what follows, I will describe a proprietary diagnostics system utilizing nanocrystals as labels and a drug delivery system using hepatitis B virus L particles.

\section{Nanocrystals In Diagnostics}

Lateral flow immunoassay is a mature technology and the fastest growing product sector in the diagnostic industry. It is also variously known as "one-step test", "bed-side test", or "dipstick". In the conventional assembly, it consists of multiple layer membranes with pre-loaded specific antibody to a disease and a detection system. The biggest advantage of this device is that it is simple and fast to use and low cost when manufactured in large scale. A well-known product 
example is the over-the-counter pregnancy test kit. The disadvantages of the current technology include (1) low sensitivity that has limited it from detecting low level of viruses or disease markers, (2) low throughput because it detects only one disease or symptom at a time, and (3) poor capability in quantitative detection, whereas diagnoses of many diseases require quantitative determination.

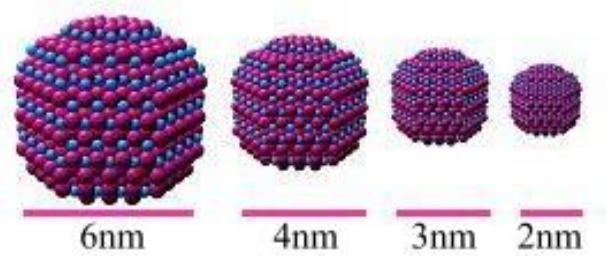

Figure 3. Samples of semiconductor nanocrystals.

Recent advances in nanotechnology have created a great product line, semiconductor nanocrystals (SCNs) or quantum dots, which are currently commercially available. SCNs have sizes equivalent to proteins $(2-6 \mathrm{~nm})$ and possess extraordinary optical properties. One such striking property is that their emission wavelength can be tuned to any chosen wavelength simply by changing their size or material composition. This property provides a sound foundation for multiplexing applications in high throughput screening. Compared to conventional dyes used in detection systems, SCNs also have many other highly desirable properties: extremely high photostability, symmetric emission (no red tail), very sharp color (FWHM $\leq 35 \mathrm{~nm}$ ), high quantum yield (as high as 90\%), and very strong fluorescence. Furthermore, all colors can be excited via a single broadband UV source, unlike conventional systems that require multiple, expensive laser excitation sources. This latter property makes the design and manufacturing of instruments using SCNs much easier. Contrary to organic dyes used by current diagnostics technologies, SCNs bleach extremely slowly with light exposure, allowing long integration times 
and high sensitivity when template material is scanned. SCNs can also be coated with a variety of biological materials, forming ideal detection systems for in vitro diagnostics (IVD).

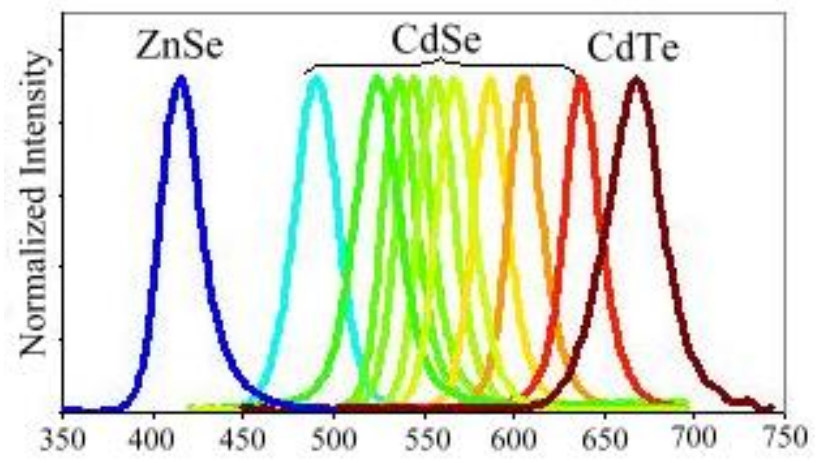

Figure 4. Size- and material-dependence of fluorescence property of semiconductor nanocrystals (SCNs), the emission wavelength is in $\mathrm{nm}$, the excitation of $\mathrm{ZnSe}$ is at $290 \mathrm{~nm}$, while the other two are at $365 \mathrm{~nm}$. ZnSe emits blue fluorescence while depending on size; CdSe emits fluorescence from green to red; CdTe emits far-red fluorescence, which is very unique and useful for biological labeling or probing.

Among many emerging companies, NanoBiotech Sdn Bhd (NBX) is exploiting these unique optical and photochemical properties of SCNs to manufacture NBX lateral flow immunoassay chips for diagnosis of infectious diseases. Strong fluorescence of the NBX chips increases the sensitivity, making quantitative detection more accurate and reliable; the multiplexing capability enables users to simultaneously detect multiple diseases leading to high throughput and dramatic reduction of cost per test; single wavelength excitation of all colors of SCNs greatly simplifies the design of signal detectors, leading to lower cost of NBX detection instrument. All in all, using SCNs in diagnostics not only empowers lateral flow immunoassay high sensitivity and accuracy, but also reduces the cost to patients and hospitals by several folds. 


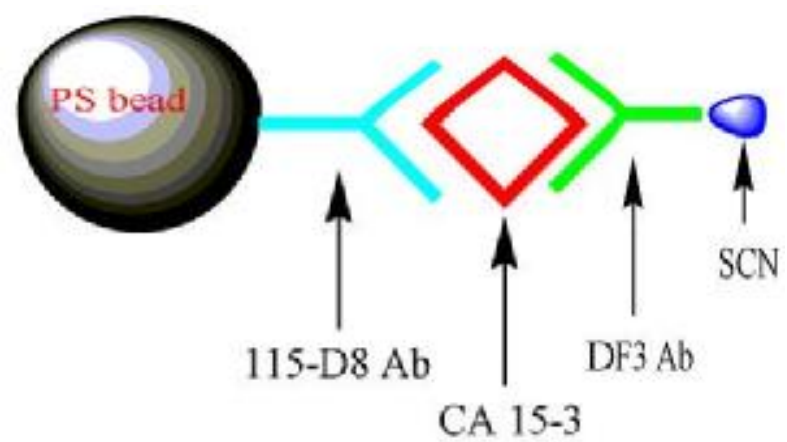

Figure 5. Cancer diagnostics using the tumor marker of CA 15-3 in a double-determinant or sandwich fluoroimmunoassay. The monoclonal antibody 115-D8, attached to a polystyrene bead, binds to the mucin-like membrane glycoprotein CA 15-3 (marker), pulling it out of solution. The SCN-labeled monoclonal antibody DF3 binding the CA 15-3 and the polystyrene beads are then subjected to FACS analysis.

In the current diagnostic industry, Total Laboratory Automation (TLA) systems have been developed and installed in most large hospitals and reference laboratories in developed countries. These large instruments are actually highly automated classical assays. The NBX systems with new concepts and new technologies can offer competitive advantages in all key aspects as an integrated diagnostic system.

Table 3. Key feature comparison between a TLA system and NanoBiotech NBX system.

\begin{tabular}{lll}
\hline Feature & TLA System & NBX System \\
\hline High sensitivity & Yes & Yes \\
High specificity & Yes & Yes \\
Networking & Yes & Yes \\
High throughput & Yes & Yes (through multiplex and fast turn over) \\
Automation & Yes & Not necessary as it is an easy one-step operation \\
Quick result & No & Yes \\
Low cost & No & Yes \\
Affordable for small clinics & No (huge and expensive) & Yes (small and low cost) \\
Small volume specimen & No & Yes \\
\hline
\end{tabular}

\section{Gene And Drug Delivery Using Hepatitis B Virus L Particles}

Hepatitis B virus (HBV) has a 3.2 kilobase-pair (Kbp) genome and is a human liver-specific 
virus. The genome harbors three overlapping envelope (env) genes in a single open reading frame, encoding small (S), medium (M) and large (L) proteins. Since 1990, hollow particles of HBV env S, M and L proteins have been produced in yeast cells using recombinant technology. The $\mathrm{S}$ and $\mathrm{M}$ particles have been safely used as immunogens in hepatitis B vaccines. ${ }^{7-9}$ The $\mathrm{L}$ particles have an average diameter of $80 \mathrm{~nm}$, consist of more than $100 \mathrm{~L}$ protein molecules embedded in a yeast endoplasmic reticulum (ER) membrane-derived phospholipid vesicle without HBV genome inside and are readily purified by ultracentrifugation. ${ }^{10-11}$ The L particles have a pre-S1 peptide, the N-terminal amino acid residues 108-119 of the L protein displayed on their surface and this peptide functions as the specific ligand for receptor binding on human hepatocytes. $^{12}$

In a recent small step forward in gene and drug delivery to human hepatocytes, Japanese and Belgian researchers have successfully tested the $\mathrm{L}$ particles, derived from the envelope of hepatitis B virus and grown in genetically engineered yeast cells, as gene transfer vehicles. ${ }^{13}$ In the first set of tests to show specificity in tissue cultures and on mouse xenografts, the subunit (pre-S1 peptide) selectively bound to human liver cells and the L particles transferred a gene for green fluorescent protein (GFP) to human liver cell carcinomas, but not other carcinomas or tissues. In a second set of tests on mouse xenografts, L particles successfully transferred the gene for human clotting factor IX (hFIX). The xenograft cells then produced significant levels of the clotting factor. 


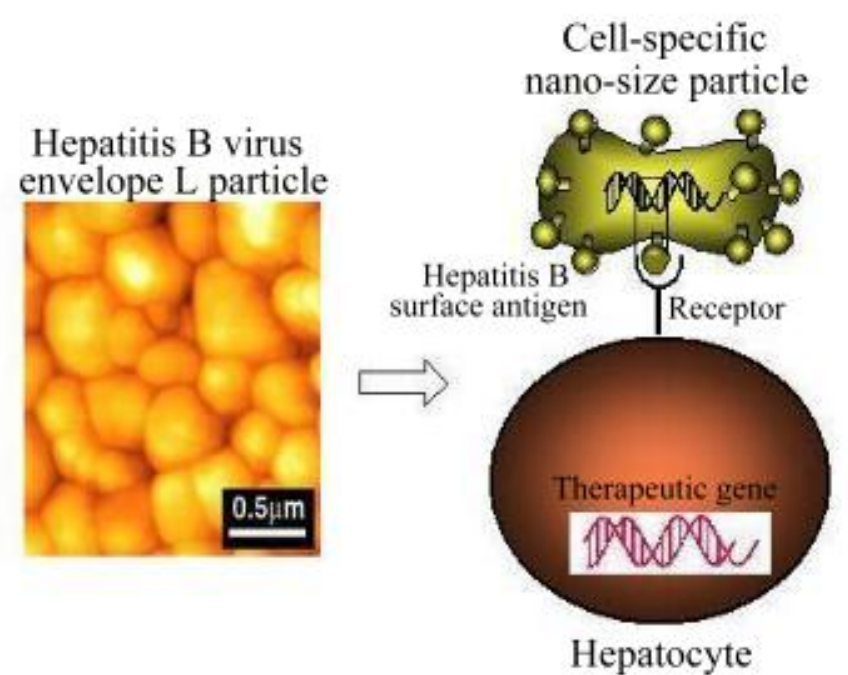

Figure 6. (Left panel) An atomic force microscopy (AFM) image of hepatitis B virus envelope L particles. (Right panel) The nano-size particle is made up of a hollow lipid vesicle that displays the hepatitis B virus attachment protein, $\mathrm{L}$ antigen, which is liver-specific.

This delivery system has a number of advantages: safety (no detectable toxicity); efficiency (high levels of expression); specificity (highly selective in binding) and large packaging load (can deliver DNA larger than $40 \mathrm{Kbp})$. A current challenge of the delivery method is that most of the world's population has antibodies against hepatitis B either from prior infection or from immunization. The application of the L particle delivery system would be limited to patients who have no antibodies against the virus. However, a possible way to overcome the limitation is to eliminate or modify the immunogenic epitopes from $\mathrm{L}$ particles or to separate $\mathrm{L}$ particles from the mutant form of hepatitis B virus. ${ }^{14}$

\section{DISCUSSIONS AND CONCLUSION}

Though the current technology has not come close to being able to shrink macro objects and organisms down to sizes of "The Incredible Shrinking Man" and "Fantastic Voyage", concurrent research and development efforts in diagnostics platforms and delivery systems utilizing 
nanotechnology and nano-size materials are breaking new grounds. In the clinical treatment of the near future, a complete molecular-based diagnosis of a disease could be accomplished by just a few antibodies, at low cost, within seconds using a desk-top or palm-size detection system, and target-specific genes or drugs could be delivered to the organs in question, even in the very early stages of the disease. ${ }^{15}$

The TLA systems of today may process 200 or more immunoassays or chemical assays per hour and can run 24 hours a day. They typically cost US\$3 million each and some systems may cost up to US\$8 millions. In computer analogy, these TLA systems are the equivalents of mainframe computers of yesteryear. If the systems can be made small (miniaturized) and cheap (affordable) like personal computers, the marketplace will find ways to use them. Many companies and research centers are actively engaged in this quest of miniaturization and affordability. The personal TLA system will likely become a commercial reality, albeit in several guises, in the next few years, and a self-monitoring market will emerge, just like personal computers (PC) and palm-held digital personal assistants (PDA) are the vade mecums of modern urban trendsetter. ${ }^{16}$

\section{ACKNOWLEDGMENTS}

The author would like to thank Professors Marinee KL Chuah and Thierry VandenDriessche of University of Leuven, Belgium for enlightening discussions of the gene and drug delivery system and for providing sketches. The author also acknowledges the help of Drs. Jianquan David Liu and Jianfu Jeffrey Wang for stimulating discussions.

\section{REFERENCES}

1. Cleaner living through nanotech. Associated Press, September 9, 2002.

2. Lim, H.A. GENETICALLY YOURS: Bioinforming, biopharming, and biofarming. World Scientific Publishing, Inc., Teaneck, New Jersey, 2002. 
3. Feynman, R. There is plenty of room at the bottom. Engr. \& Sci., 1960, 22-36.

4. Asimov, I. Fantastic Voyage. Bantam Books, New York, 1966.

5. Crichton, M. Prey. HarperCollins Publishers Inc., New York, 2003.

6. Service, R. Nanofiber fabric unveiled. Science, 2003, 611, 1.

7. Kuroda, S., Itoh, Y., Miyazaki, T., Otaka-Imai, S., and Fujisawa, Y. Efficient expression of genetically engineered hepatitis B virus surface antigen P31 proteins in yeast. Gene, 1989, 78, 297-308.

8. Fujisawa, Y., Kuroda, S., Van Eerd, P.M., Schellekens, H., and Kakimura, A. Protective efficiency of a novel hepatitis B vaccine consisting of $\mathrm{M}$ (pre-S2+S) protein particles (a third generation vaccine). Vaccine, 1990, 8, 192-198.

9. Kuroda. S., Fujisawa, Y., Lino, S., Akahane, Y., and Suzuki H. Induction of protection level of anti-pre-S2 antibodies in humans immunized with a novel hepatitis B vaccine consisting of $\mathrm{M}$ (pre-S2+S) protein particles (a third generation vaccine). Vaccine, 1991, 9, 163-169.

10. Kuroda, S., Otaka, S., Miyazaki, T., Nakao, M., and Fujisawa, Y. Hepatitis B virus envelope L protein particles, synthesis and assembly in Saccharomyces cerevisiae, purification and characterization. J. Biol. Chem., 1992, 267, 1953-1961.

11. Yamada, T., Iwabuki, H., Kanno, T., Tanaka, H., Kawai, T., Fukuda, H., Kondo, A., Seno, M., Tanizawa, K., and Kuroda, S. Physicochemical and immunological characterization of hepatitis B virus envelope particles exclusively consisting of the entire L (pre-S1+pre-S2+S) protein. Vaccine, 2001, 19, 3154-3163.

12. Le Seyec, J., Chouteau, P., Cannie, I., Guguen-Guillouzo, C., and Gripon, P. Infection process of hepatitis B virus depends on the presence of a defined sequence in the pre-S1 domain. J. Virol., 1999, 73, 2052-2057.

13. Yamada, T., Iwasaki, Y., Tada, H., Iwabuki, H., Chuah, M., VandenDriessche, T., Fukuda, H., Kondo, A., Ueda, M., Seno, M., Tanizawa, K., and Kuroda, S. Nanoparticles for the delivery of genes and drugs to human hepatocytes. Nature Biotechnology, 2003, 21(8), 885-890.

14. Lawrence, D. Nanotechnology takes another step forward. Lancet, 2003, 362, 48.

15. Gardner, E. Ultimate analysis. Bio-IT World, 2003, Nov 14.

16. Lim, H.A. CHANGE: in business, corporate governance, education, scandals, technology and warfare. EN Publishing, Inc., Santa Clara, California, 2003. 